\title{
Extended Spectrum Beta-Lactamases Among Hospitalized Patients in Surgery Wards, Ilam, Iran
}

Nourkhoda Sadeghifard ${ }^{1}$, Sobhan Ghafourian' ${ }^{1}$, Zamberi bin Sekawi², Vasantha Kumari Neela², Ali Hematian¹, Iraj Pakzad ${ }^{1}$, Elham Abouali Galehdari ${ }^{1}$ and Reza Mohebi $^{1 *}$

${ }^{1}$ Department of Medical Microbiology, Ilam university of Medical Sciences, Iran

${ }^{2}$ Department of Medical Microbiology, University Putra Malaysia, Malaysia

\begin{abstract}
Objectives of this study were to study the molecular epidemiology of ESBL-producing Klebsiella pneumoniae in selective hospitals in Iran, to determine the prevalence of TEM, SHV and CTX-M genes responsible for ESBL production among ESBL-producing K. pneumoniae, to investigate the susceptibility of Klebsiellae spp producing ESBLs towards non-beta-lactam antibiotics, all in different seasons. Clinical isolates of $K$. pneumoniae were identified during Mar. 2007 to Apr. 2008 in Ilam hospitals, the province in west of Iran. All isolates were found in surgery wards. ESBL activity was first evaluated using the standard disc diffusion test for cephalosporins and monobactam, then using the double-disc synergy test between cephalosporins and clavulanate. PCR assay had done for ESBLs genes detection. The results showed sixteen K.pneumoniae were identified by chemical methods. No resistance had occurred among K.pneumoniae toward non-beta-lactam antibiotics. BlaSHV was dominant gene responsible for ESBLs production while just one blaTEM along with blaSHV were found. BlaCTX-M was not responsible for ESBLs production in our study. $37.5 \%$ K.pneumoniae producing ESBLs in surgery ward in west of Iran must be considerable and need to further study in different part of hospital in Ilam and in Iran. Strict antibiotic policy should be adopted in hospitals to estimate the impact of higher resistance in bacteria and to take steps for reducing these resistances.
\end{abstract}

Keywords: K.pneumoniae, surgery ward, Iran

\section{Introduction}

ESBLs are known as extended-spectrum because they are able to hydrolyze a broader spectrum of beta-lactam antibiotics than the simple parent beta-lactamases from which they are derived. Such ESBLs have also the ability to inactivate beta-lactam antibiotics containing an oxyimino-group such as oxyimino-cephalosporins (eg; ceftazidime, ceftriaxone, cefotaxime) as well as oxyiminomonobactam [1,2]. Furthermore, they are not active against cephamycins and carbapenems. Generally, they are inhibited by beta-lactamase-inhibitors such as clavulanate and tazobactam. ESBLs have been found in a wide range of Gram-negative rods. However, the vast majority of strains expressing these enzymes belong to the Enterobacteriaceae family [1]. K.pneumonia seems to remain as the major ESBL-producer [3]. The strong selective pressure for the use of beta-lactam drugs exerted on ESBL producer strains may lead to the selection of strains that hyperproduce ESBL, to the emergence of strains expressing different types of ESBLs, to the selection of complex mutant enzymes with inhibitor resistant phenotype or porin alteration which lead to the development of resistance to cephamycins and other antimicrobials $[4,5,6]$. The plasmids that harbor genes encoding ESBLs frequently contain other genes encoding mechanisms of resistance to aminoglycoside and cotrimoxazole [7]. Quinolone resistance is frequently found in ESBL producer strains; although, the mechanism of co resistance is not clear [8]. Objectives of this study were to study the molecular epidemiology of ESBL-producing Klebsiella pneumoniae in selective hospitals in Iran, to determine the prevalence of TEM, SHV and CTX-M genes responsible for ESBL production among ESBLproducing Klebsiellae pneumoniae, to investigate the susceptibility of Klebsiellae spp producing ESBLs towards non-beta-lactam antibiotics, all in different seasons.

\section{Methods}

\section{Bacterial isolates}

Clinical isolates of K. pneumoniae were identified during the period
March 2007 to April 2008 in in Ilam hospitals, the province in west of Iran. All isolates were found in surgery wards.

\section{Identification of Klebsiella pneumoniae}

Gram stained in Modified Preston-Morrel Method, Oxidase and Catalase. Media: SIM, Simon Citrate, MR-VP, Lysine Iron Agar, Kligler Agar, Phenylalanin Agar, Urea Agar, Malonate, Blood Agar, Maccanky Agar [9] were used in our study.

\section{Antibiotic testing}

Susceptibilities to antimicrobial agents were determined by the antibiotic disc diffusion method. ESBL activity was first evaluated using the standard disc diffusion test for cephalosporins and monobactam, then using the double-disc synergy test between cephalosporins and clavulanate [10].

Escherichia coli ATCC 25922 was used as an ESBL-negative reference strain, and K. pneumoniae ATCC 700603 was used as an ESBL-positive reference strain.

\section{PCR amplification of blaTEM, SHV and CTX-M}

DNA extraction was carried out using a rapid alkaline lysis method [3]. The oligonuclotide primers used for the PCR assays were: blaTEM (F: 5-GAGTATCAACATTTCCGTGTC-3, R: 5-TAATCAGTGAG-

*Corresponding author: Reza Mohebi, Department of Medical Microbiology, Ilam university of Medical Sciences, Iran, E-mail: Sobhan.ghafurian@gmail.com

Received November 09, 2010; Accepted December 28, 2010; Published January 05, 2011

Citation: Sadeghifard N, Ghafourian S, bin Sekawi Z, Neela VK, Hematian A, et al. (2011) Extended Spectrum Beta-Lactamases Among Hospitalized Patients in Surgery Wards, Ilam, Iran. J Microbial Biochem Technol 3: 018-020. doi:10.4172/1948-5948.1000044

Copyright: (c) 2011 Sadeghifard N, et al. This is an open-access article distributed under the terms of the Creative Commons Attribution License, which permits unrestricted use, distribution, and reproduction in any medium, provided the original author and source are credited. 
GCACCTTCTC-3), blaSHV (F:5-AAGATCCACTATCGCCCAGCAG-3,R:5 ATTCAGTTCCGTTTCC CAGCGG-3) [11] and blaCTXM (F:5-ACGCTGTTGTT AGGAAGTG-3, R:5-TTGAGGCTGGGTGAAGT-3) [12].

\section{Results}

Sixteen isolates of K.pneumoniae were identified by chemical methods. They were tested against $3^{\text {rd }}$ generation of cephalosporins and aztreonam. Results analyzed by SPSS. The results allocated $37.5 \%$ resistance to ceftazidime (Ca) $(30 \mu \mathrm{g})$, Cefteriaxone (Ci) $(30 \mu \mathrm{g})$,

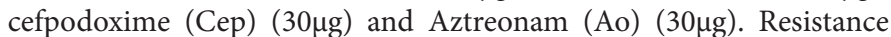
toward cefotaxime $(\mathrm{Ce})(30 \mu \mathrm{g})$ was $50 \%$ (Table 1). K.pneumoniae resistant to aztreonam and one or more $3^{\text {rd }}$ generation of cephalosprins were suspected to produce ESBLs. Confirming stage performed by using of Ceftazidime alone (30ug) versus ceftazidime/clavulanic (Cac) $(30 / 10 \mu \mathrm{g})$, cefotaxime $(30 \mu \mathrm{g})$ alone versus cefotaxime /clavulanic acid (Cec) $(30 / 10 \mu \mathrm{g})$ and cefpodoxim alone versus cefpodoxim /clavulanic acid (Cepc) $(30 / 10 \mu g)$. These results showed all K.pneumoniae that were suspected to produce ESBLs were confirmed by Cac and Cepc, while didn't observe confirmation by Cec. Regardless of the zone diameters, a $>5 \mathrm{~mm}$ increase in a zone diameter for an antimicrobial agent tested in combination with clavulanic acid versus its zone size when tested alone, indicated a probable ESBL production [13]. Resistance to non-betalactam antibiotics including: amikacin (Ak) $(30 \mu \mathrm{g})$, cotrimoxazol (Co) $(30 \mu \mathrm{g})$ ciprofloxacin (Cf) $(30 \mu \mathrm{g})$, imipenem (I) $(30 \mu \mathrm{g})$ didn't observe. BlaSHV was dominant gene responsible for ESBLs production while just one blaTEM along with blaSHV were found. BlaCTX-M was not responsible for ESBLs production in our study (Figure 1).

\section{Discussion}

The development of extended-spectrum cephalosporins in the early

\begin{tabular}{|c|c|c|c|c|c|c|}
\hline & K.pneumoniae & $\mathbf{C a}$ & $\mathbf{C e}$ & $\mathbf{C i}$ & $\mathbf{C e p}$ & Ao \\
\hline \multirow{2}{*}{ Spring } & 3 & 1 & 0 & 1 & 0 & 0 \\
\hline \multirow{2}{*}{ Summer } & $(18.75 \%)$ & $(33.33 \%)$ & 0 & $(33.33)$ & 0 & \\
\hline \multirow{2}{*}{ Fall } & $(25 \%)$ & $(50 \%)$ & 3 & 1 & 2 & 2 \\
& $(31.25 \%)$ & $(75 \%)$ & $(25 \%)$ & $(50 \%)$ & $(50 \%)$ \\
\hline \multirow{2}{*}{ Winter } & 4 & 4 & 2 & 3 & 3 \\
& $(25 \%)$ & $(25 \%)$ & 1 & 2 & 1 & 1 \\
\hline \multirow{2}{*}{ Total } & $\mathbf{1 6}$ & $\mathbf{6}$ & $\mathbf{6} \%)$ & $(50 \%)$ & $(25 \%)$ & $(25 \%)$ \\
\hline
\end{tabular}

Table 1: Screening stage for detection of K.pnemoniae producing ESBLs from admitted patients in Surgery wards in llam hospitals.

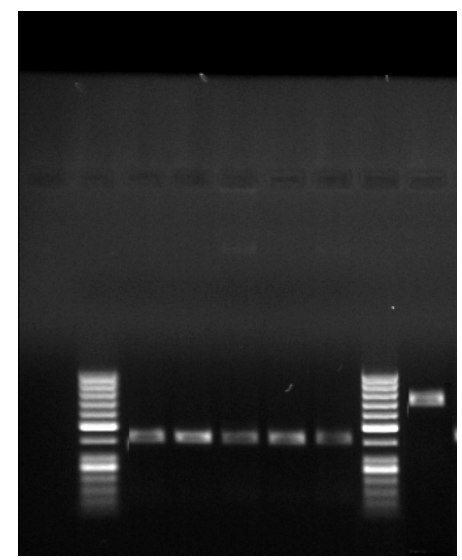

Figure 1: Right to left: Marker=50bp, five blaSHV, Marker=50bp, blaTEM. 1980s was regarded as major addition to our therapeutic armamentarium in the fight against beta-lactamase-mediated bacterial resistance [14] This data obtained from clinical samples of K. pneumoniae, shows high antibiotic resistance. Worldwide resistance to antibiotics has increased in Klebsiella [15].

Carbapenems are the drugs of choice for many infections caused by gram positive and gram-negative bacteria [16]. Imipenem, cotrimoxazol, ciprofloxacine and amikacin were found to be the most effective antibiotics against K.pneumoniae producing ESBLs in this study. Nowadays, ESBLs is considered a problem among the hospitalized patients throughout the world. The prevalence of ESBLs among the clinical isolates, which is rapidly changing over time, varies greatly and geographically worldwide. Patients suffering from infections caused by ESBL- producing organisms are at increasing risks of treatment failures with broad-spectrum beta-lactam antibiotics. In a survey, one hundred and sixty-eight clinical isolates of K.pneumoniae were collected during September 2006 to February 2007 from three general hospitals of Tehran in Iran .The most isolates of K.pneumoniae were from urine ( $\mathrm{n}=$ $82)$, respiratory tract $(n=21)$ and blood $(n=16)$ specimens. $69 \%$ from One hundred and sixty eight clinical isolates were positive and fifty-one isolates $(31 \%)$ were negative for ESBLs production [11]. By the early 1990 s, 25 to $35 \%$ of nosocomially acquired K.pneumoniae isolates were ESBL producing in France (Marty et al., 1998). In 1988, isolates of $K$. pneumoniae containing SHV-2 were reported from China (Jacoby et al., 1988). Several outbreaks of infections with ESBL-producing Klebsiella spp. have been reported from South Africa Cotton, et al. [17], but no national surveillance figures have been published. However, it has been reported that $36.1 \%$ of K.pneumoniae isolates collected in a single South African hospital in 1998 and 1999 were ESBL producers (Bell et al., 2002). Different percentage of ESBLs had reported in different parts of the world. Our study showed 37.5\% of K.pneumoniae had ESBLs in surgery ward in west of Iran. Thus, it must be considerable further study in different part of Iran. Cefteriaxone resistance in fall had the highest percentage of resistant due to $3^{\text {rd }}$ generation of cephalosporins in surgery ward (80\%) and K.pneumoniae producing ESBLs more occurred in fall, too. The dominant gene responsible for ESBLs production was BlaSHV while the lowest frequency of ESBLs gene had observed for BlaCTX-M. Studies of molecular epidemiology of these resistance genes can also be used for comparison with genes already isolated from other parts in Iran and of the world.

In conclusion, strict antibiotic policy should be adopted in hospitals to estimate the impact of higher resistance in bacteria and to take steps for reducing these resistances.

\section{Acknowledgment}

Ilam University of medical sciences provided partial support for the laboratory studies and interpretation. We hereby declare that it has not been previously or concurrently submitted for any other journals. All others are agreeing with this statement.

\section{References}

1. Bradford PA (2001) Extended spectrum beta-lactamases in the 21st century: characterization, epidemiology and the detection of this important resistance threat. Clin Microbiol Rev 14: 933-951.

2. Paterson DL, Bonomo RA (2005) Extended-spectrum beta-lactamases: a clinical update. Clin Microbiol Rev 18: 657-686.

3. Winokur PL, Brueggemann A, DeSalvo DL, et al. (2000) Animal and human multidrug - resistant, cephalosporin-resistant Salmonella isolates expressing a plasmid-mediated CMY-2 AmpC beta-lactamase. Antimicrob Agents Chemother 44: 2777-2783.

4. Bradford PA,Cherubin CE, Idemyor V, Rasmussen BA, Bush K(1994) Multiply resistant Klebsiella pneumoniae from two Chicago hospitals: identification of 
Citation: Sadeghifard N, Ghafourian S, bin Sekawi Z, Neela VK, Hematian A, et al. (2011) Extended Spectrum Beta-Lactamases Among Hospitalized Patients in Surgery Wards, Ilam, Iran. J Microbial Biochem Technol 3: 018-020. doi:10.4172/1948-5948.1000044

the extended-spectrum TEM-12 and TEM-10 ceftazidime-hydrolyzing betalactamases in a single isolates. Antimicrob Agents Chemother 38: 761-766.

5. Pangon B, Bizet C, Bure A, et al. (1989) In vivo selection of a cephamycin resistant, porin deficient mutant of Klebsiella pneumoniae producing a TEM-3beta- lactamase. J Infect Dis 159:1005-1006.

6. Sirot D, Chanal C, Henquell C, Labia R, Sirot J, et al. (1994) Clinical isolates of Escherichia.coli producing multiple TEM mutants resistant to beta-lactamase inhibitors. Journal of Antimicrobial Chemotherapy 33: 1117-26.

7. Villa L, Pezzella C, Tosini F, Visca P, Petrucca A, et al. (2000) Multipleantibiotic resistance mediated by structurally related IncL/M plasmids carrying an extended spectrum beta-lactamase gene and class 1 integron. Antimicrob Agents Chemother 44: 2911-2914.

8. Paterson DL, Mulazimoglu L, Casellas JM, et al. (2000) Epidemiology of ciprofloxacin resistance and its relationship to extended-spectrum betalactamase production in Kelbsiella pneumoniae isolates causing bacteremia. Clin Infect Dis 30: 473-478.

9. Macfaddin JF(1999) Biochemical tests for identification of medical bacteria. $3^{\text {rd }}$ dedition Philadelphia lippincott Williams and Wilkinson.

10. National Committee for Clinical Laboratory Standards. Performance Standards for Antimicrobial Susceptibility Testing: (2004) Fourteenth Informational Supplement M100-S14. NCCLS, Wayne, PA, USA
11. Shahcheraghi F, Moezi H, Feizabadi MM (2007) Distribution of tem and SHV B-lactamase genes among klebsiella pneumoniae strains isolated from patients in Tehran. 13: BR247-250.

12. Mansouri M, Ramazanzadeh R (2009) Spearead of extended spectrum betalactamases producing E.coli clinical isolates in sanandaj hospital. Journal of biological sciences 9: 362-366.

13. National Committee for Clinical Laboratory Standards (1999) Performance standards for antimicrobial susceptibility testing; ninth informational supplement. Wayne PennsysIvania NCCLS document M100-S9 Vol. 19 No. 1.

14. Bush K (2002) The impact of beta-lactamases on the development of nove antimicrobial agents. Curr Opin Investig Drugs 3: 1284-1290.

15. Shannon K, Phillips I (1980) The effects on beta-lactam susceptibility of phenotypic induction and genotypic derepression of beta-lactamase synthesis. Journal of Antimicrobial Chemotherapy 18: 15-22.

16. Yu Y, Zhou W, Chen Y, Ding Y, Ma Y (2002) Epidemiological and antibiotic resistant study on extended-spectrum beta-lactamase-producing Escherichia coli and Klebsiella pneumoniae in Zhejiang Province. Chin Med J 115: 14791482.

17. Cotton, Wasserman MFE, Pieper $\mathrm{CH}$, Theron DC, van Tubbergh D, et al (2000) Invasive disease due to extended spectrum beta-lactamase-producing Klebsiella pneumoniae in a neonatal unit: the possible role of cockroaches. $J$ Hosp Infect 44:13-17. 